\title{
Erratum: Realization of the Dicke Model Using Cavity-Assisted Raman Transitions [Phys. Rev. Lett. 113, 020408 (2014)]
}

\author{
Markus P. Baden, Kyle J. Arnold, Arne L. Grimsmo, Scott Parkins, and Murray D. Barrett \\ (Received 23 February 2017; published 9 May 2017)
}

DOI: 10.1103/PhysRevLett.118.199901

We would like to withdraw our Letter [1] concerning the realization of a Dicke model within a high finesse cavity. In recent experiments that investigated a Dicke-model simulation with imbalanced driving [2], inconsistencies between the more recent results and the demonstration in our Letter [1] were encountered. This prompted us to reevaluate the earlier work. Re-analysis of the experimental data led us to conclude that the work of Baden et al. [1] was not a realization of a Dicke-model phase transition as claimed but simply a single beam effect: a single cavity-assisted Raman transition transferring population from one state to the other. This was confirmed by a series of independent experiments. Reconsideration of the applicable theory showed that the copropagating geometry used in the original demonstration [1] cannot be mapped to a Dicke-model Hamiltonian and that a counterpropagating configuration must be used as demonstrated in Ref. [2]. A detailed account of the theory and results for the counterpropagating geometry will appear elsewhere.

[1] M. P. Baden, K. J. Arnold, A. L. Grimsmo, A. S. Parkins, and M. D. Barrett, Realization of the Dicke model using cavity-assisted Raman transitions, Phys. Rev. Lett. 113, 020408 (2014).

[2] Z. Zhiqiang, C. H. Lee, R. Kumar, K. J. Arnold, S. J. Masson, A. S. Parkins, and M. D. Barrett, Non-equilibrium phase transition in a spin one Dicke model, Optica 4, 424 (2017). 